\title{
Cepheid Diameters from Optical Interferometry: The NPOI Survey
}

\author{
Tyler E. Nordgren, M. E. Germain, J. J. Sudol
}

U.S. Naval Observatory, Astrometry Dept., NPOI, P.O. Box 1149, Flagstaff $A Z$ 86001-1149, USA

R. B. Hindsley, J. T. Armstrong

Remote Sensing Division, Naval Research Laboratory, Code 7210, Washington $D C$ 20375, USA

Arsen R. Hajian

U.S. Naval Observatory, Astrometry Dept., 3450 Massachusetts Avenue $N W$, Washington $D C$ 20392, USA

\begin{abstract}
We present linear radii for four Cepheid variable stars, spanning a pulsation period range from 3 to $11 \mathrm{~d}$, measured with the Navy Prototype Optical Interferometer (NPOI). We compare these radii to those found using traditional indirect methods, and to various period-radius relations found in the literature.
\end{abstract}

\section{Introduction}

Accurate stellar radii are important for the study of Cepheid structure, mass and pulsation properties. Direct diameter measurement of bright, nearby Cepheids allows for comparison to diameters found by indirect and/or theoretical methods. Since there will always be Cepheids too small or too faint for direct measurement, this comparison is crucial for the diameter estimation of ever more distant Cepheids.

\section{The NPOI}

The NPOI has conducted routine interferometric observations on three baselines for the past three years. In that time a variety of scientific programs have been underway, one of which involves the measurement of angular diameters of giant and supergiant stars. Comparison of these measurements with angular diameters measured at other interferometers and with diameters estimated using the infrared flux method show agreement at the $1 \%$ level (Nordgren et al. 1999). 


\section{The Cepheid Survey}

With its current longest baseline of $38 \mathrm{~m}$ and a magnitude limit of $m_{V} \sim 5$ the NPOI can partially resolve four Cepheids: $\delta$ Cephei, $\zeta$ Geminorum, $\eta$ Aquilae and $\alpha$ Ursae Minoris. With the exception of $\zeta \mathrm{Gem}$, each Cepheid was observed over at least two full pulsation cycles in the last two years. Polaris was observed over 10 nights, $\delta$ Cephei for 17, $\eta$ Aquilae for 11 and $\zeta$ Geminorum for 4 . We use the same analysis technique described in Nordgren et al. (1999) to derive a mean uniform-disk angular diameter and error about that mean from the multiple observations for each Cepheid. From the uniform-disk diameter a limbdarkened diameter is derived based on atmosphere models for a star of the same average effective temperature, surface gravity and spectral class of the Cepheid (Nordgren et al. 1999). Table 1 lists the measured uniform-disk angular diameter, $\theta_{\mathrm{U}}$, the derived limb-darkened angular diameter, $\theta_{\mathrm{L}}$, the Hipparcos parallax distance, $\mathrm{d}_{\mathrm{H}}$, and linear radius, $R$, for each Cepheid.

Table 1. The NPOI Cepheid Survey

\begin{tabular}{lccll}
\hline Cepheid & $\begin{array}{c}\theta_{\mathrm{U}} \\
{[\mathrm{mas}]}\end{array}$ & $\begin{array}{c}\theta_{\mathrm{L}} \\
{[\mathrm{mas}]}\end{array}$ & $\begin{array}{l}d_{\mathrm{H}^{a}} \\
{[\mathrm{pc}]}\end{array}$ & $\begin{array}{l}R \\
{\left[R_{\odot}\right]}\end{array}$ \\
\hline$\alpha \mathrm{UMi}$ & $3.14 \pm 0.02$ & $3.28 \pm 0.02$ & $132 \pm 8$ & $47 \pm 3$ \\
$\delta \mathrm{Cep}$ & $1.46 \pm 0.02$ & $1.52 \pm 0.02$ & $301 \pm 53$ & $51 \pm 9$ \\
$\eta$ Aql & $1.65 \pm 0.04$ & $1.73 \pm 0.05$ & $360 \pm 120$ & $66 \pm 22$ \\
$\zeta$ Gem & $1.48 \pm 0.08$ & $1.55 \pm 0.09$ & $358 \pm 100$ & $60 \pm 18$ \\
\hline
\end{tabular}

${ }^{a}$ Hipparcos parallaxes from Perryman et al. (1997)

\section{Pulsation Parallax}

The large errors in the linear radii in Table 1 are due to the large uncertainties in the parallax. In an attempt to derive the distance directly from the NPOI data, the pulsation phase for each individual observation (of which there are approximately 50 per Cepheid) is found. The individual observed diameters are averaged in phase, choosing bins that yield approximately half a dozen mean diameters over a pulsation phase while minimizing the scatter in phase for each mean. This averaging is done for both $\delta$ Cep and $\eta \mathrm{Aql}$ (the amplitude of pulsation for $\alpha \mathrm{UMi}$ is far below the precision of the NPOI diameter measurements, while $\zeta$ Gem has only been observed over a small range in its pulsation). Using radial velocity observations from Shane (1958) the linear diameter displacement as a function of phase is determined. Equation 1 shows the relation between angular and linear diameter:

$$
D_{\mathrm{o}}+\Delta D(t)=0.001 \times d \times \theta(t)
$$

where $D_{\mathrm{o}}$ is the mean stellar diameter in $\mathrm{AU}, \Delta D(t)$ and $\theta(t)$ are the linear displacement and angular diameter (in milliarcseconds) at a given phase, and $d$ is the distance in parsecs. Figure 1 a plots equation 1 for $\delta$ Cephei. As is sometimes done with Baade-Wesselink and/or surface brightness methods of 

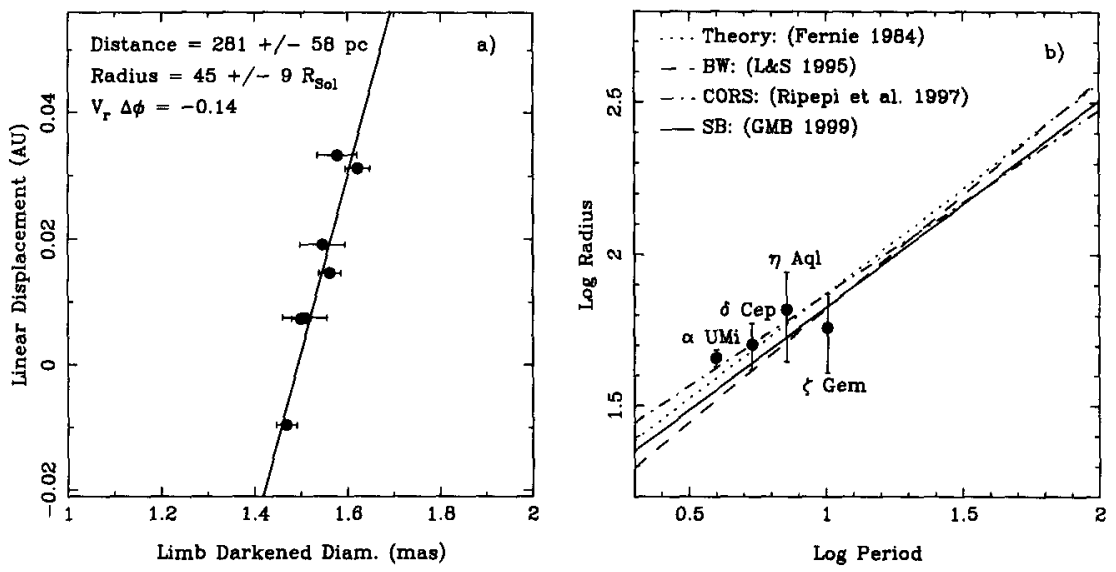

Figure 1. (a) The linear displacement versus angular size relation for $\delta$ Cephei from equation 1. (b) Period-radius relations where those shown from the literature are: theory (Fernie 1984), Baade-Wesselink (Laney \& Stobie 1995), CORS (Ripepi et al. 1997) and surface brightness relations (Gieren, Moffett, \& Barnes 1999).

determining Cepheid diameters and distances, the phase of the radial velocity displacement data is varied in order to find the phase shift which minimizes the scatter about the straight line implied by equation 1 (Ivanov 1984; Moffett \& Barnes 1987). The minimum scatter is found for a radial velocity phase shift of $-0.14(+0.09,-0.06)$. The distance and radius for $\delta$ Cephei implied by Figure $1 \mathrm{a}$ is $281 \pm 58 \mathrm{pc}$ and $45 \pm 9 R_{\odot}$, in excellent agreement with the data in Table 1 . The distance and radius for $\eta$ Aquilae are $222 \pm 73 \mathrm{pc}$ and $40 \pm 14 R_{\odot}$, which are also in agreement with the values in Table 1 . At the present time, the precision of the distance as determined using the pulsation parallax is of comparable precision to the Hipparcos parallax distance.

\section{Period-Radius Relations and the Literature}

Figure $1 \mathrm{~b}$ shows the period-radius relation for a variety of indirect diameter methods from the literature. Included are the four Cepheids measured by the NPOI with linear radii determined using Hipparcos distances. At the present time our Cepheid radii are consistent with all $P-R$ relationships and so we are unable to discriminate between competing models.

As an example of the accuracy of the NPOI observations, Table 2 lists diameters for $\delta$ Cephei found in the literature, along with the two methods for determining a mean radius using the NPOI data. Within the error bars, both NPOI measurements are identical and in excellent agreement with values from the literature. 
Table 2. Comparison of $\delta$ Cephei Radii

\begin{tabular}{ll}
\hline$R / R_{\odot}$ & Method and Source \\
\hline $52 \pm 10$ & GI2T $\langle\theta\rangle$ (Mourard et al. 1997) and $d_{\mathrm{H}}=301 \pm 53 \mathrm{pc}$ \\
$51 \pm 9$ & NPOI $\langle\theta\rangle$ and $d_{\mathrm{H}}=301 \pm 53 \mathrm{pc}$ \\
$45 \pm 9$ & NPOI Pulsation parallax $\Rightarrow d=281 \pm 58 \mathrm{pc}$ \\
$43 \pm 1$ & Baade-Wesselink method (Turner 1988) \\
$43 \pm 3$ & Cluster fitting to CV Mon (Turner 1988) \\
$41 \pm 2$ & Surface brightness (Moffett and Barnes 1987) \\
$40 \pm 3$ & Inverted B-W method (Ivanov 1984) \\
$37 \pm 4$ & Infrared flux method (Fernley, Skillen, \& Jameson 1989) \\
\hline
\end{tabular}

\section{Conclusion}

The NPOI is able to measure diameters of Cepheid variables which are in excellent agreement with those in the literature. Given the great uncertainty in the distances we are unable, at the current time, to discriminate between different methods of indirectly measuring Cepheid diameters.

Acknowledgments. We are grateful to Don Fernie for help in determining current ephemerides for $\alpha \mathrm{UMi}$ and $\delta$ Cephei. Use was made of the Galactic Cepheid Database maintained by the David Dunlap Observatory.

\section{References}

Fernie, J. D. 1984, ApJ, 282, 641

Fernley, J. A., Skillen, I., \& Jameson, R. F. 1989, MNRAS, 237, 947

Gieren, W. P., Moffett, T. J., \& Barnes III, T. G. 1999, ApJ, 512, 553

Ivanov, G. R. 1984, Ap\&SS, 105, 369

Laney, C. D. \& Stobie, R. S. 1995, MNRAS, 274, 337

Moffett, T. J. \& Barnes III, T. G. 1987, ApJ, 323, 280

Mourard, D., Bonneau, D., Koechlin, L., Labeyrie, A., Morand, F., Stee, P., Tallon-Bosc, I., \& Vakili, F. 1997, A\&A, 317, 789

Nordgren, T. E., Germain, M. E., Benson, J. A., Mozurkewich, D., Sudol, J. J., Elias II, N. M., Hajian, A. R., White, N. M., Hutter, D. J., Johnston, K. J., Gauss, F. S., Armstrong, J. T., Pauls, T. A., \& Rickard, L. J. 1999, $\mathrm{AJ}$, in press

Perryman, M. A. C., Lindegren, L., Kovalevsky, J., Høg, E., Bastian, U., et al. 1997, A\& A, 323, L49

Ripepi, V., Barone, F., Milano, L., \& Russo, G. 1997, A\&A, 318, 797

Shane, W. W. 1958, ApJ, 127, 573

Turner, D. G. 1988, AJ, 96, 1565 


\section{Discussion}

Nancy Evans: $\eta \mathrm{Aql}$ has a fairly bright companion which has never been resolved. (No orbital velocity variation has been seen, so the orbit is probably fairly wide.) Do you see any sign of the companion in your observations?

Tyler Nordgren: No. We have looked, but see no sign in our data of a companion. Part of this, however, may be due to the fact that we have very poor sensitivity in the blue part of the spectrum. As a result, we just may not be sensitive to such a companion.

Siobahn Morgan: Moskalik \& Ogloza's poster (p. 237 in these proceedings) and my poster presented at the Cape Town conference (IAU Coll. No. 155) propose that Polaris is an overtone mode Cepheid. The main result for this is to move it in the $P-R$ diagram by about $\log P=0.15$ to longer period, which would put it in line with some of the other relations on the $P-R$ diagram. Also, can you clarify whether Polaris has stopped pulsating?

Tyler Nordgren: The difference in position on the $P-R$ diagram due to Polaris being a first overtone pulsator is a good point. I had not considered it but I will now. What I'd meant to say is that given the precision of our diameter observations, we have not detected the pulsation. Recent work, by Fernie for instance, indicates that not only has the amplitude of Polaris' pulsation stopped decreasing, but, in fact, it may now be increasing. 